Type: Research Paper

Section: Optoelectronics

\title{
Optical code division multiplexing in the design of encoded fiber Bragg grating sensors
}

\author{
C. A. Triana ${ }^{1,2 *}$, D. Pastor ${ }^{1}$, M. Varón ${ }^{2}$ \\ 1. Optical \& Quantum Communications Group, iTEAM Research Institute, Univ. Politécnica de Valencia, Camino \\ de Vera s/n, 46022 Valencia, Spain. \\ 2. High frequency electronics and telecommunications research group CMUN, Universidad Nacional de Colombia, \\ Cra 30 \# 45-06, 111321 Bogotá, Colombia. \\ (*) E-mail: catrianai@unal.edu.co
}

Received 02/10/2015 Accepted: 23/02/2016

DOI: $10.7149 /$ OPA.49.1.17

\begin{abstract}
:
The design of spectrally encoded fiber Bragg grating (FBG) sensors is proposed in order to incorporate more information in the optical measurement system. This information can provide full distinction between sensors operating at the same wavelength range. The encoded sensors are inspired by Optical Code Division Multiplexing (OCDM) techniques, specifically, we used Optical Orthogonal Codes (OOC) in order to define the spectral shape of the FBG sensors, providing them with a unique spectral signature. As a result we demonstrated the detection and tracking of the proposed sensors which allows effective measurement of temperature or strain even under overlapping conditions. The interrogation of the proposed encoded sensors could be performed easily and in real time through the autocorrelation product between the reflected spectrum and each sensor codeword. A simple addition to this identification method is implemented in order to remove any error in the interrogation process. Furthermore, a simulation of the proposed sensors under overlapping conditions is performed in order to test the distinction capability of the system. Finally the manufacturing and measurement of the sensors is described.
\end{abstract}

Key words: Fiber Bragg gratings, FBG, Optical sensing, Encoded sensors.

\section{REFERENCES AND LINKS}

[1] A. Kersey, M. Davis, H. Patrick, M. Leblanc, K. Koo, C. Askins, M. Putnam, and E. Friebele, Fiber grating sensors, Lightwave Technology, Journal of 15, 1442-1463 (1997).

http://dx.doi.org/10.1109/50.618377

[2] B. Lee, Review of the present status of optical fiber sensors, Optical Fiber Technology 9, 57 - 79 (2003). http://dx.doi.org/10.1016/S1068-5200(02)00527-8

[3] A. Kersey, A. Dandridge, and M. Davis, Low-crosstalk code-division multiplexed interferometric array, Electronics Letters 28, 351-352 (1992).

http://dx.doi.org/10.1049/el:19920219 
[4] H. Lee, Multiple fiber Bragg grating sensor system using code-division multiple access, Appl. Opt. 41, 5245-5248 (2002).

http://dx.doi.org/10.1364/A0.41.005245

[5] K. Koo, A. Tveten, and S. Vohra, Dense wavelength division multiplexing of fibre Bragg grating sensors using CDMA, Electronics Letters 35, 165-167 (1999).

http://dx.doi.org/10.1049/el:19990135

[6] J. Ko, Y. Kim, and C.-S. Park, Fiber Bragg grating sensor network based on code division multiple access using a reflective semiconductor optical amplifier, Microwave and Optical Technology Letters 52, 378$381(2010)$.

http://dx.doi.org/10.1002/mop.24913

[7] A. Taiwo, S. Seyedzadeh, R. Sahbudin, M. Yaacob, M. Mokhtar, and S. Taiwo, Performance comparison of OCDMA codes for quasi-distributed fiber vibration sensing, in Photonics (ICP), 2013 IEEE 4th International Conference on, (2013), pp. 111-113.

[8] H.-C. Cheng, C.-H. Wu, C.-C. Yang, and Y.-T. Chang, Wavelength division multiplexing/spectral amplitude coding applications in fiber vibration sensor systems, Sensors Journal, IEEE 11, 2518-2526 (2011). http://dx.doi.org/10.1109//SEN.2011.2128308

[9] M. Fernandez-Vallejo, D. Ardanaz, and M. Lopez-Amo, Optimization of the available spectrum of a WDM sensors network using a mode-locked laser, Lightwave Technology, Journal of 33, 4627-4631 (2015).

[10] L. Chen, Optical code-division multiple-access enabled by fiber Bragg grating technology, in Optical Science and Engineering, CRC Press, pp. 111-164 (2005).

http://dx.doi.org/10.1201/9781420026610.ch4

[11] J. Chen, H. Jiang, T. Liu, and X. Fu, Wavelength detection in FBG sensor networks using least squares support vector regression, Journal of Optics 16 (2014).

http://dx.doi.org/10.1088/2040-8978/16/4/045402

[12] T. Erdogan, Fiber grating spectra, Lightwave Technology, Journal of 15, 1277-1294 (1997).

[13] M. Best, A. Brouwer, F. MacWilliams, A. Odlyzko, and N. Sloane, Bounds for binary codes of length less than 25, Information Theory, IEEE Transactions on 24, 81-93 (1978). http://dx.doi.org/10.1109/TIT.1978.1055827

[14] F. Chung, J. Salehi, and V. Wei, Optical orthogonal codes: design, analysis and applications, Information Theory, IEEE Transactions on 35, 595-604 (1989).

[15] S. Ayotte, M. Rochette, J. Magne, L. Rusch, and S. LaRochelle, Experimental verification and capacity prediction of FE-OCDMA using superimposed FBG, Lightwave Technology, Journal of 23, 724-731 (2005).

[16] D. Meghavoryan and A. Daryan, Superimposed fiber Bragg grating simulation by the method of single expression for optical CDMA systems, Photonics Technology Letters, IEEE 15, 1546-1548 (2003). http://dx.doi.org/10.1109/TIT.1978.1055827

[17] R. Baños, D. Pastor, W. Amaya, and V. Garcia-Munoz, Chromatic dispersion compensation and coherent direct-sequence OCDMA operation on a single super structured FBG, Opt. Express 20, 13966-13976 (2012). http://dx.doi.org/10.1364/OE.20.013966

[18] R. Baños, V. Garcia-Munoz, D. Pastor, and W. Amaya, Rectangular global envelope super structured FBGs for multiband coherent OCDMA, Photonics Technology Letters, IEEE 25, 512-514 (2013).

http://dx.doi.org/10.1109/LPT.2013.2242880 


\section{Introduction}

Fiber Bragg grating sensors (FBGs) have been widely accepted, in both, the academia and the industry due to their excellent performance characteristics as sensors. Their sensitivity, reduced size and electromagnetic immunity are between their outstanding features. This optical device acts as a filter that reflects a narrow set of wavelengths from the incident light spectrum. Their working principle relays on the shift of the reflected wavelength according to the environmental conditions affecting the optical fiber region surrounding the grating (classically, temperature and strain) [1].

The use of multiplexing schemes is common in optical sensing networks; these methods aim to increase the number of operational sensors in the available spectrum. Given the working principle of FBG sensors (wavelength shifts) the most suitable, and used, multiplexing methodology is the wavelength domain multiplexing (WDM) [2], in which each sensor is constrained to a wavelength range. In this sense, the maximum amount of sensors corresponds to the total incident spectrum divided into the assigned operation range for each sensor. Even though WDM is the most popular scheme, there have been proposed several alternatives using the time domain multiplexing (TDM) method, however, these approaches represent more difficult detection schemes as they require synchronization in the signal acquisition.

Multiplexing optical FBG sensors with these architectures implies the specification of a working range to each sensor. Indeed, as each sensor is not always intended to neither measure the same variables nor in the same range, the WDM spectral division is usually performed considering a safeguard common to all the sensors. This represents a problem of efficiency since some regions of the spectrum are unexploited.

Other approaches to interrogate FBG sensors using concepts from OCDMA have been previously presented, for example, in references [3-6] it is proposed the modulation of a broadband optical source by a pseudorandom binary sequence (PRBS). Then, electronic autocorrelation is performed in order to distinguish in the time domain the signals from each sensor in the network. In this scheme, the electronic PRBS en/decoding is able to distinguish a high number of sensors with very low crosstalk but, it can be limited by phase noise due to mixing of time coincident pulses from different sensors [3]. In [7], OCDMA was also proposed as a classical multiplexing technique to share the transmission fiber to perform vibration measurements and, in [8] OCDMA-SAC (Spectral Amplitude Coding) was proposed and demonstrated for the multiplexed transmission of vibration signals. However, in both cases the FBG arrays are static in both, reflectivity and Bragg wavelength and thus not acting as sensors. Another approach is presented in [9], where the measurement system is based on a Mode-Locked Laser created by an array of paired FBGs, thus, the sensing information is encoded in the resonant wavelength of each cavity. Identification of the sensing signals is accomplished by wavelength and time signal measurements.

In this work, we propose the application of Optical Code Division Multiplexing (OCDM) techniques to the design of FBG sensors. The objective is to change the spectral shape of the FBG sensors providing them with more information, and consequently, enhancing the discerning process between sensors.

From this approach we take advantage of two direct features of OCDM techniques [10]. Firstly, the demodulation of the proposed encoded FBG sensors is implemented as the autocorrelation product between each sensor's codeword and the acquired amplitude spectrum. In this way the spectral position from each sensor is obtained unequivocally without requiring additional computational methods [11]. And secondly, from the concept of orthogonal codification; wavelength overlapping between two encoded sensors is possible without affecting the measurement of each sensors signal.

This document is organized as follows: section 2 describes the selection and characteristics of the orthogonal codes employed to shape spectrally the proposed encoded FBG sensors and introduces the concept of wavelength identification for these sensors. In section 3 the proposed sensors are simulated in order to evaluate their behavior and their main design features, along with their effect over the wavelength identification process. Section 4 explains the manufacturing process of the super imposed FBG devices and shows the experimental results carried out to validate the theory. Finally, some conclusions are derived in section 5 . 


\section{Spectrally encoded FBG sensors}

A simple fiber Bragg grating sensor consists on a periodical structure inscribed inside the optical fiber's core that reflects a single wavelength $\lambda_{B}=2 \eta_{e f f} \Lambda$ [12], where $\Lambda$ is the spatial period of the grating and $\eta_{e f f}$ is the effective refractive index; the changes in the environment of the fiber affect both parameters leading to a wavelength shift.

One of the concerns taken into account when designing serial FBG sensors networks is the overlapping between two adjacent sensors. When two contiguous sensors are shifted in such a way that they cross each other in the spectrum, the interrogation instrument (which usually detects the maximum peak of the reflected signal) is not able to associate each detected wavelength to the correct sensor in the network.

The proposed spectrally encoded FBGs provide a unique spectral signature to each sensor in order to expand the information contained in each one. Thus, in an overlapping scenario it is possible to identify independently the spectral shape of each sensor, using its own signature. Enabling complete tracking of the reflected wavelength from each sensor in the system.

Encoded sensors' design consists on devices with multiple spectral bands, inspired in concepts from OCDM, concretely, the use of Optical Orthogonal Codes (OOC) in order to perform a codification in the spectral domain.

\section{2. a. Selection of Optical Orthogonal Codes}

Optical orthogonal codes (OOCs) $[13,14]$ have been deeply studied in the field of OCDM communications, where, a unique codeword is assigned to each user in the network in order to give access to multiple users to the same physical communications channel at the same time. Thus, the information from each user is spread out by the OOC in the time domain and individually decoded at the end of the communications link. In an analogous way, we are interested in providing each sensor with a distinctive spectral shape, in such a way that, every pair of adjacent sensors in the network display a unique spectral shape. Moreover, when the pair of sensors get closer and overlapped; there is always a way to identify the spectral shape corresponding to each one of the sensors (due to their orthogonal characteristics). In order to achieve complete distinction between $\mathrm{k}$ sensors, each one must contain $\mathrm{k}+1$ spectral bands allowing that, in any wavelength shifting scenario at least one band of the super structure in the interrogated sensor is free of spectral overlapping from other neighbor sensors.
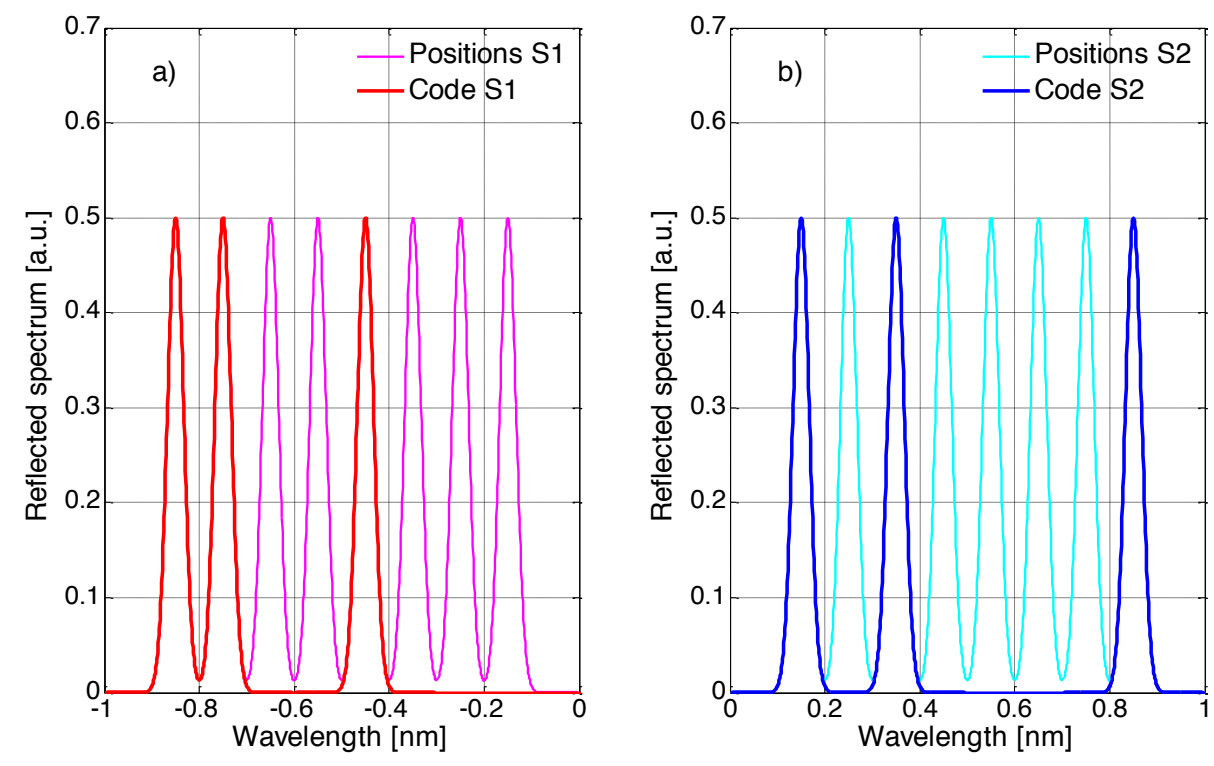

Fig.1. In a) it is depicted the codeword S1 = [11001000], along with the available positions corresponding to the zeros in the code. b) Shows the same plot for the codeword S2 = [10100001]. 
In the encoding of FBG sensors we are interested in simple and short orthogonal codes. These codes are generated by the combinatorial method as described in [14]. We used the following codewords: $S 1=[11001000] ; S 2=[10100001]$, to represent two orthogonally encoded sensors. This means that each sensor features 8 equally spaced spectral slots, with three reflection sub-bands in the positions corresponding to the ones. The spectral shape of the two codewords S1 and S2 are represented in Fig 1 a) and b) respectively, it is depicted the shape of each sensor and the available positions of the code.

\section{2. b. Demodulation approach}

The interrogation technique for this kind of sensors is related directly with the concept of O0Cs. The orthogonality of the codes implies that the autocorrelation product (AC) of the codeword with itself gives a unitary peak (namely the auto-correlation peak ACP) as a result, while the product between the codeword and other possible combination of codewords produces a lower level signal, known as the cross-correlation product (XC). This concept allows us to identify the spectral position of the sensor by evaluating the autocorrelation product between each codeword and the measured reflected spectrum from the FBG sensors. Fig. 2 shows the individual autocorrelation product for sensors S1 in a) and S2 in b), each plot represents the ACP and the cross-correlation product obtained from the spectra.
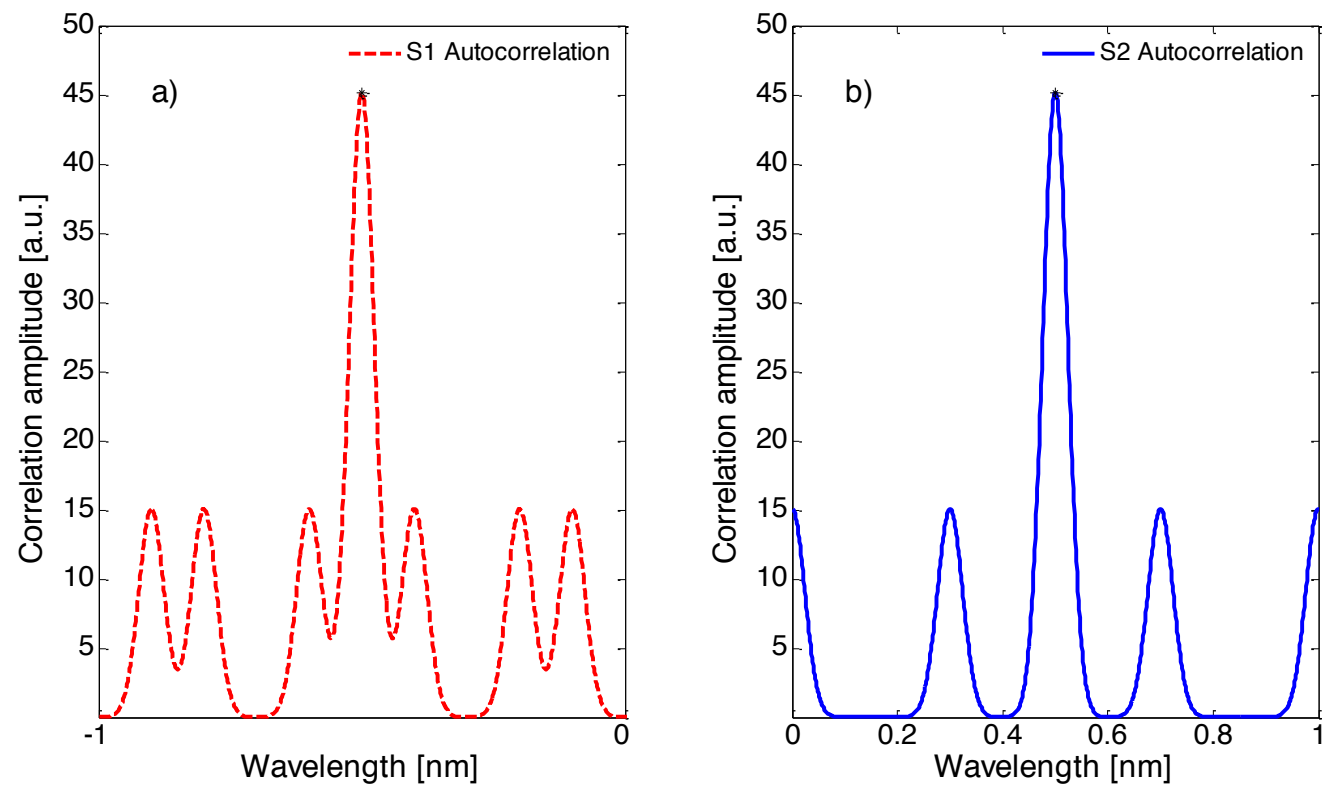

Fig. 2. The left plot, a) shows the autocorrelation product from the codeword S1, while in the right plot, b), it is depicted the autocorrelation corresponding to the sensor S2.

When two or more $(K)$ spectrally neighbor sensors are shifted due to changes in the environment, its reflected spectrum $R(\lambda)$ can be written as shown in equation (1):

$$
R(\lambda)=\sum_{j=1}^{K} \sum_{i=-N / 2}^{N / 2} c_{j}(i) * R * g\left(\lambda-\lambda_{B j}-\Delta \lambda_{B j}-(\delta \lambda * i)\right)
$$

where $g(\lambda)$ is the normalized spectral shape of the sub-bands into the sensors, $\lambda_{B j}$ and $\Delta \lambda_{B j}$ are the design Bragg wavelengths and their shift (due to environmental fluctuations), $\delta \lambda$ is the spectral separation between sub-bands inside each coded sensor (spectral slot) and $c_{j}(i)$ is a binary value $\{0,1\}$ corresponding to the $j$-th encoded sensor and $i$-th sub-band into the code. After the spectral measurement, the Identification Function (IF) for each sensor can be obtained evaluating numerically the following expression: 


$$
I F_{j}\left(\lambda^{\prime}\right)=\int\left(\sum_{i=-N / 2}^{N / 2} c_{j}(i) * g\left(\lambda-\lambda^{\prime}-(\delta \lambda * i)\right)\right) R(\lambda) d \lambda
$$

This process is performed for each encoder $j$ and in the case of no overlapping the maximum $I F_{j}\left(\lambda^{\prime}\right)$ provides a peak located at the exact position $\lambda_{B j}+\Delta \lambda_{B j}$ (i.e the AC peak of code $j$ ). For a general case of partial or total overlapping the $I F_{j}\left(\lambda^{\prime}\right)$ function will include a summation of the autocorrelation (AC) of the code $j$ and the Cross-Correlation (XC) of the code $j$ with the remaining overlapped codes. In Fig. 3 it is depicted the complete reflected spectrum of two neighbor sensors, S1 and S2, in overlapping conditions and its corresponding autocorrelation. In Fig. 3 a), the first sensor (S1) is represented with a red continuous line while sensor (S2) corresponds to the blue dashed line; the total reflected spectrum is represented in black. In $3 \mathrm{~b}$ ) it is presented the result from the identification function for each sensor, each peak represents the central spectral location of sensor S1 (red) and S2 (blue).
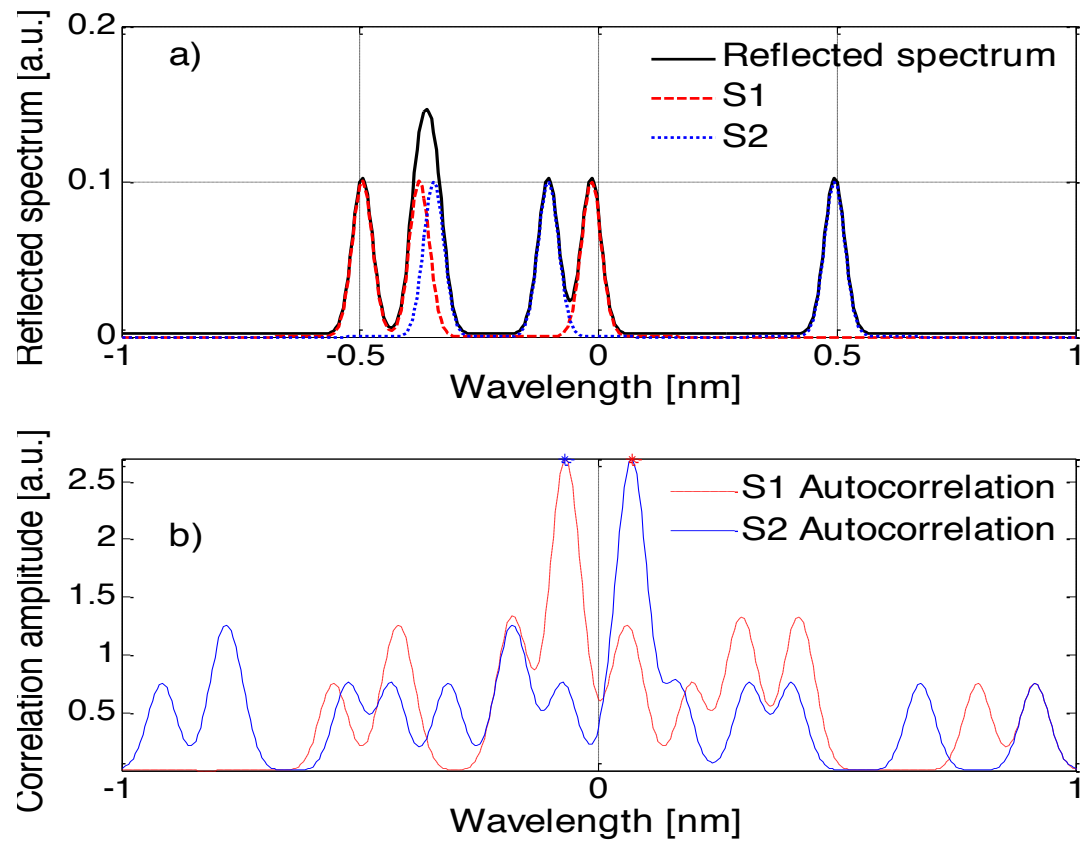

Fig. 3. In a) the total reflected spectrum and the two interfering encoded FBG sensors are depicted. b) Shows the autocorrelation identification function for each sensor.

As mentioned before, the autocorrelation identification function is computed over the overall measured spectrum, so it implies that the cross-correlation product added to the AC desired peak is going to affect the sensor central position estimation (if only the AC peak position or maximum is taken). Nevertheless, the proposed encoding scheme offers more information that can be extracted in order to remove this error in the identification process. This is carried out by implementing a simple two step identification approach. This consists in an additional step that allows the confirmation of the exact position of each sensor; parting from the central position found by the identification function (2) it is possible to ignore the overlapped sub-bands of the sensors and recalculate the center of the sensor from the unperturbed sub-bands. With this procedure we can remove the error from interfering sensors in the identification process as we are neglecting the error source, fully exploiting their orthogonality.

\section{Simulation results}

As a proof of the concept, computer simulations were carried out in order to evaluate the overall behavior of the encoded sensors system in terms of tracking accuracy for each sensor under overlapping conditions as well as the influence of the design parameters over the tracking results. The demodulation algorithm based in 
the autocorrelation product is capable to follow the shift of each sensor independently even when their subbands get completely overlapped. The main parameters in this simulation are: Reflectivity $=10 \%$, distance between slots $\delta \lambda=0.12 \mathrm{~nm}$, spectral width of the sub-bands $=0.05 \mathrm{~nm}$.

The trajectory shown in Fig. 4 a) corresponds to the first sensor S1, positioned initially at $-0.5 \mathrm{~nm}$ and following a linear path of $1 \mathrm{~nm}$ until $0.5 \mathrm{~nm}$. The second sensor S2 follows a trajectory in the opposed direction from $0.5 \mathrm{~nm}$ to $-0.5 \mathrm{~nm}$ as shown in Fig. $4 \mathrm{~b}$ ). These figures show the reference ideal path for each sensor with a dashed black line and, a continuous line describing the tracked trajectory obtained with the autocorrelation algorithm. In the insets, a zoomed region of the error is illustrated. The maximum error obtained for both sensors is $\pm 5 \mathrm{pm}$.
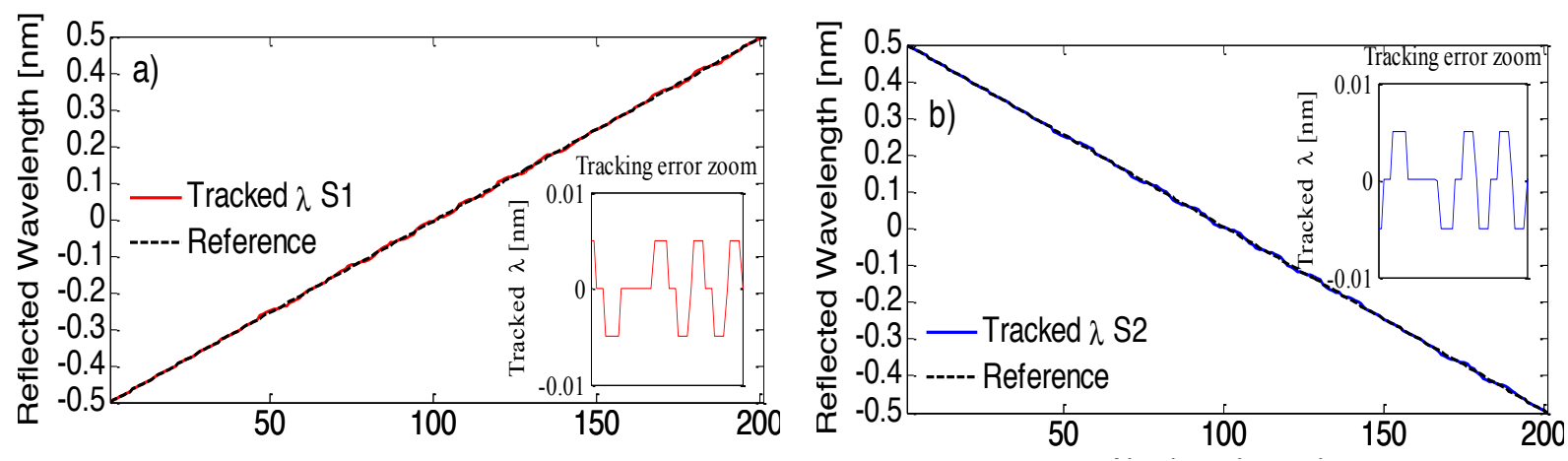

Fig. 4. Wavelength tracking of two neighbor sensors crossing each other spectrally, a) and b) show the tracking result and the reference path for each sensor respectively. In the insets it is shown the obtained error.

As mentioned before, the obtained error is caused by the interference of a cross-correlation (XC) lobe adding to the autocorrelation peak (AC). With the obtained error of $\pm 5 \mathrm{pm}$ the temperature error is of about $\pm 0.5^{\circ} \mathrm{C}$. The amount of error in the identification process depends on the ratio XC/AC determined by the number and amplitude of the XC wings, which in this case (2 sensors of 3 sub-bands) are of about $33 \%$ of the AC peak.

In order to reduce this error, the two-steps identification approach was implemented numerically without significant increment of the computation cost. This approach leads to the complete reduction of the measurement error due to CDMA multiplexing between the two sensors. In a practical system the measurement error will be reduced to the minimum feasible error given by the resolution and sweeping parameters of the instrumentation (Optical Spectrum Analyzer or any other commercial interrogator) like in the non-overlapping standard sensor case.

Regarding the shape parameters of the encoded sensors, some tests were performed in order to evaluate their influence over the identification process. Initially, the influence of the reflectivity of the sensors was evaluated for the two identification procedures; the reflectivity was modified from $10 \%$ to $100 \%$ in a serial configuration, which means that the second sensor receives the incident spectrum affected by the portions reflected by the first sensor. Fig. 5 depicts the maximum detected error for each sensor with the two proposed identification methodologies. The maximum error for the simple autocorrelation method was measured to be nearly $0.005 \mathrm{~nm}$ in all the cases. This error is completely eliminated with the two-steps identification method under low reflectivity regime. When the reflectivity is greater than $70 \%$, this method also reports error values of $\sim 0.005 \mathrm{~nm}$ for the second sensor. This is because the second sensor does not receive enough energy from the incident signal and therefore the error increases.

Another important parameter is the ratio between the slots distance $(\delta \lambda)$ and the width of each reflected band $(W)$. As we want to design compact spectrally sensors the distance between sub-bands was settled to $\delta \lambda=$ $0.12 \mathrm{~nm}$, which means that the total spectral length of the sensors (with 8 sub-bands) is $0.96 \mathrm{~nm}$. A good behavior of the system is obtained when the ratio $\delta \lambda / W$ is greater than 2 , as demonstrated in fig 6 , where the error in the detection algorithms is plotted as a function of the width of the sensors' sub-bands. There is an evident increment on the measured error for broader reflection sub-bands; as mentioned before the detection error depends on the interferences produced by the cross-correlation (XC) signals over the auto-correlation 
(AC) ones. As the sub-bands are broadening these wings also are; leading to more interference between the signals.

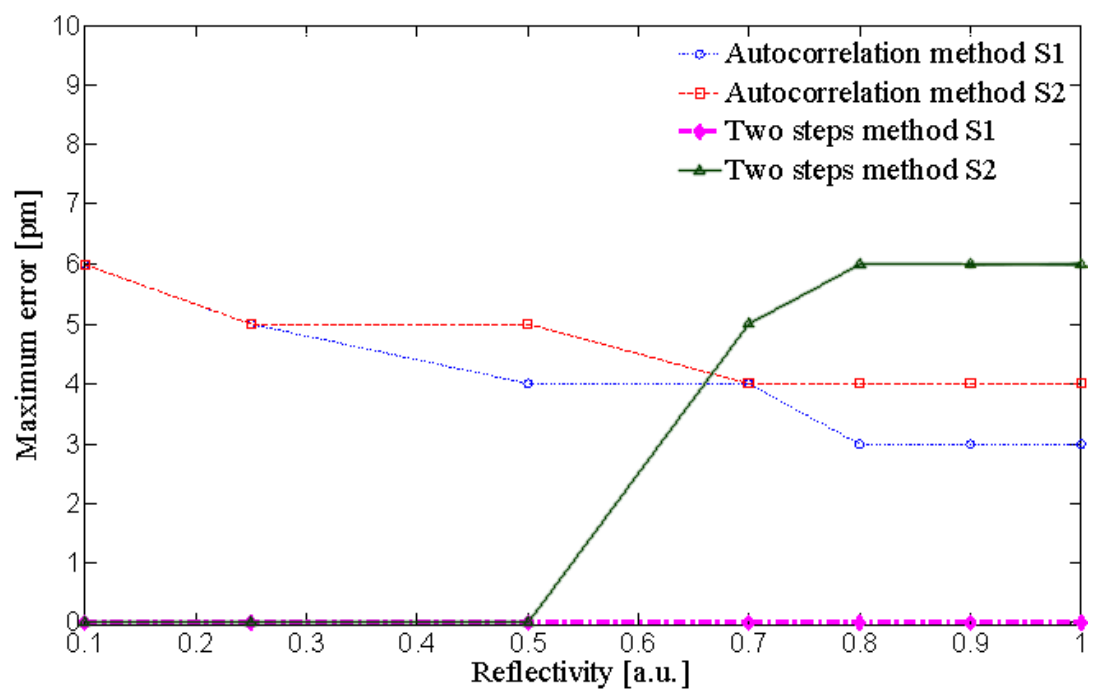

Fig. 5. Influence of the sensors' reflectivity over the measured detection error for each identification procedure.

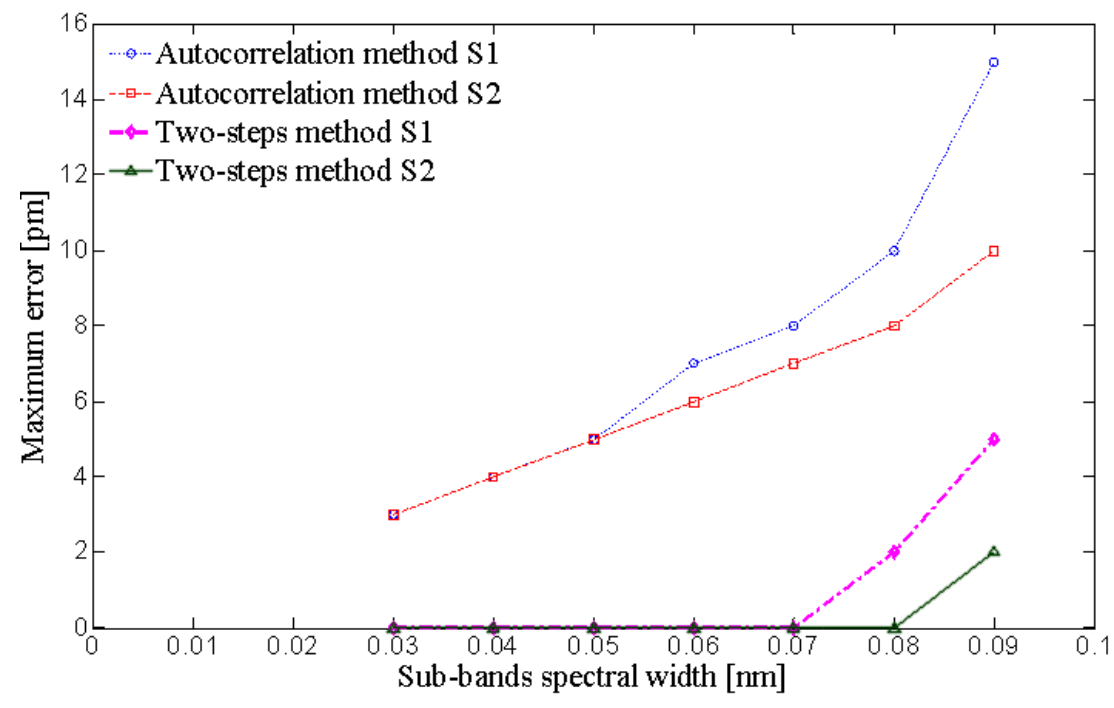

Fig. 6. Effect of the sub-bands' width over the measured detection error for each identification procedure.

\section{Manufacturing and experimental setup}

The manufacture of the proposed encoding FBG sensors could be easily carried out from the concept of SuperImposed FBGs as demonstrated for OCDMA communications systems in [15, 16]. A SI-FBG is a device in which multiple gratings have been constructed in the same location of the fiber's core. The resulting device will display a superposition of multiple reflection bands at different wavelengths. As each band is written at the same location in the optical fiber's core, their wavelength shift due to environmental effects will be the same. The proposed encoded sensors were designed employing the Discrete Layer Peeling (DLP) synthesis method, starting from the objective described in Fig. 1 and fixing the maximum reflectivity to 25\%. Manufacturing was performed by a Ultra-Violet (UV) laser beam exposition after a Phase Mask (PM) to obtain the Bragg pattern 
[17]. UV beam was focused before the PM up to $\sim 40 \mu \mathrm{m}$. Fig. 7 represents schematically the point by point manufacturing process.

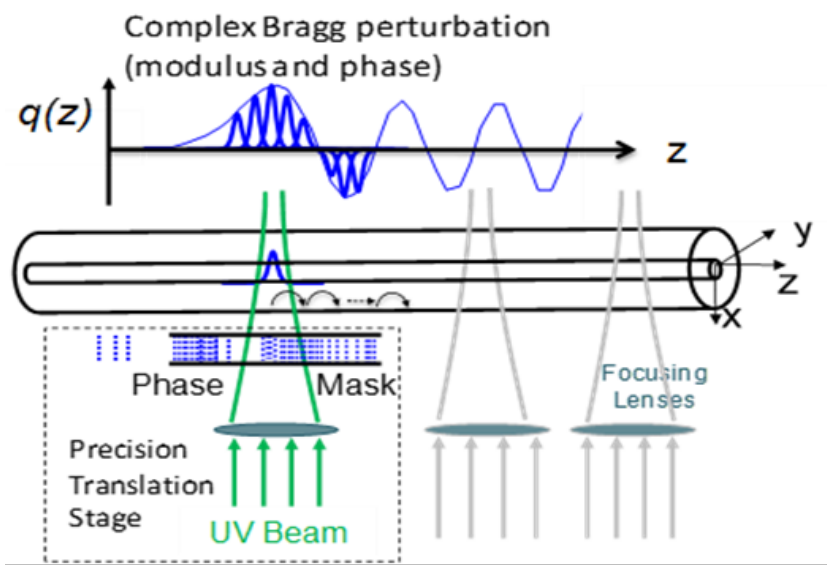

Fig. 7. Super Imposed fiber Bragg grating manufacturing process

The amplitude and phase control of each sample of $q(z)$ was achieved by a double UV exposition of $500 \mathrm{~ms}$ over the same z-position, only changing the relative phase of the Bragg period between them by the proper PM versus fibre displacement. In this way, the averaged UV flux is constant and the averaged value of the refractive index remains unaltered [18].

The spectral characterization of the manufactured encoded sensors is depicted in figs. 8 and 9 for sensors codewords S1 and S2 respectively, a very good agreement between the design and measured spectrum was obtained. Comparison parameters between the manufactured sensors and the design are summarized in table 1.

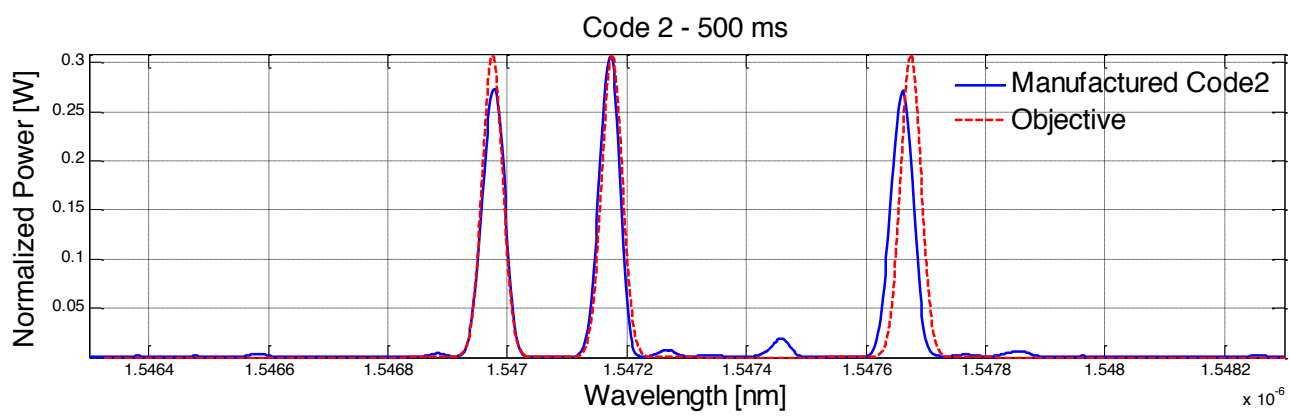

Code 2 - $500 \mathrm{~ms}$

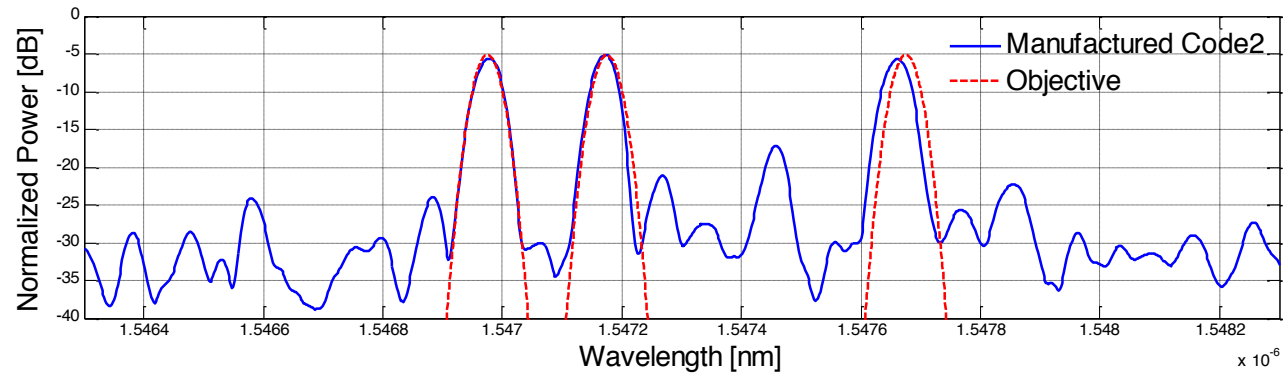

Fig. 8. Linear and logarithmic spectrum for sensor S1, in red dashed line it is represented the design objective while blue solid line represents the measured spectrum. 
Code $1-500 \mathrm{mS}$

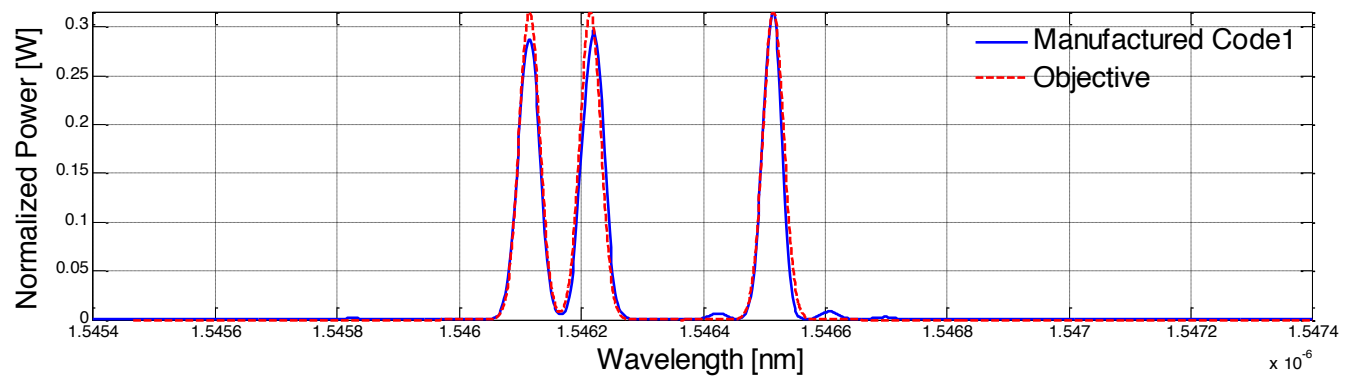

Code 1 - $500 \mathrm{mS}$

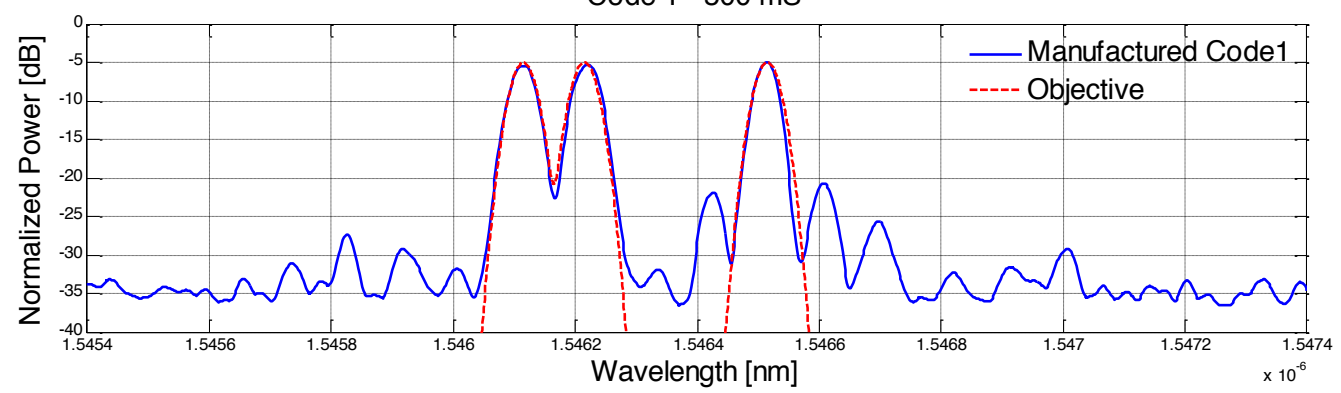

Fig. 9. Linear and logarithmic spectrum for sensor S2, in red dashed line it is represented the design objective while blue solid line represents the measured spectrum.

TABLE 1. Designed and measured features of the manufactured encoded sensors.

\begin{tabular}{|c|c|c|c|c|c|c|}
\hline \hline & $\begin{array}{c}\text { Average } \\
\text { reflectivity }\end{array}$ & \multicolumn{3}{|c|}{$\begin{array}{c}\text { Sub-bands' linewidth } \\
\text { [pm] }\end{array}$} & \multicolumn{2}{c|}{$\begin{array}{c}\text { Sub-bands' distance } \\
\text { [pm] }\end{array}$} \\
\hline \hline Designed S1 & $25 \%$ & 40 & 40 & 40 & 100 & 300 \\
\hline Sensor Codeword 1 & $31.5 \%$ & 40.8 & 42.8 & 37.76 & 104 & 294 \\
\hline Designed S2 & $25 \%$ & 40 & 40 & 40 & 200 & 500 \\
\hline Sensor Codeword 2 & $30.7 \%$ & 40.3 & 39.8 & 39.3 & 194 & 490 \\
\hline \hline
\end{tabular}

Regarding the experimental validation of the encoded FBG sensors, reflectivity is the more important design parameter, depending on the architecture used in the sensing system (i.e. Serial or parallel configuration. See Fig. 10). In the case of encoded FBG sensors (as in any standard sensor network), if we consider a serial arrangement of the different sensors along the same optical fiber at different sensing positions, then low reflectivity regime must be considered for each sensor in order to let incident optical signal reaching all the designed sensors even if they are overlapped. Parallel configuration is also feasible, deploying different optical paths for each sensor through passive splitting components (1xN star couplers). Although sharing the spectrum in the same way as the serial array, in this case the reflectivity of the sensors can be maximized being the insertion losses of the splitting element the limiting factor.

In Fig. 10 the use of the attenuator allows to vary the reflectivity of one of the sensors in order to measure different scenarios with the same sensors. The measurements were made with an OSA (10 pm resolution) in a range of $3 \mathrm{~nm}$ around $1548 \mathrm{~nm}$, acquiring 1000 pts of the spectral data, i.e. $3 \mathrm{pm}$ sampling. Temperature of the sensors was controlled and one of the sensors was stretched in order to overlap completely with the static second sensor. Fig. 11 shows a single readout of the experiment in which sensors are interfering while the identification of their central position is performed. 

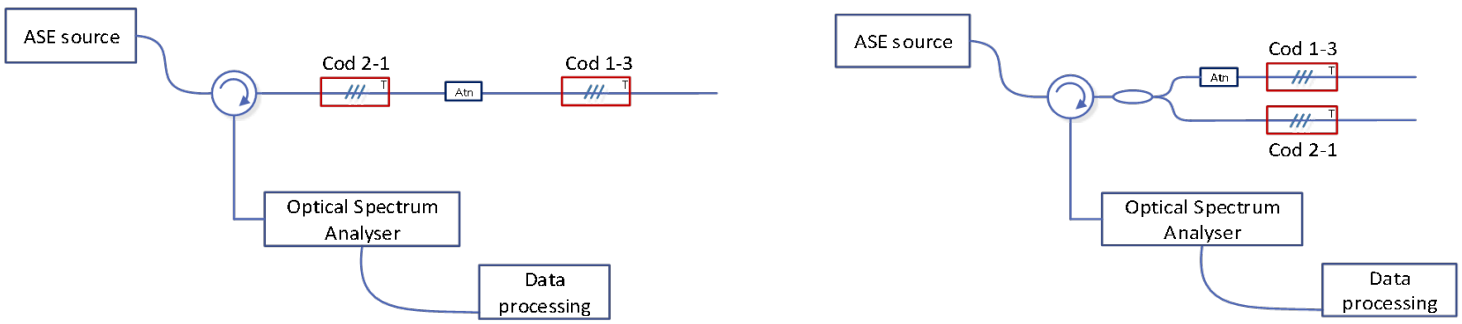

Fig. 10. Experimental setup employed in the validation of the encoded FBG sensors. In the left plot is described the serial configuration in which the reflectivity of the sensors affect the performance of the system. The plot in the right shows the parallel configuration where reflectivity of the sensors could be enhanced.

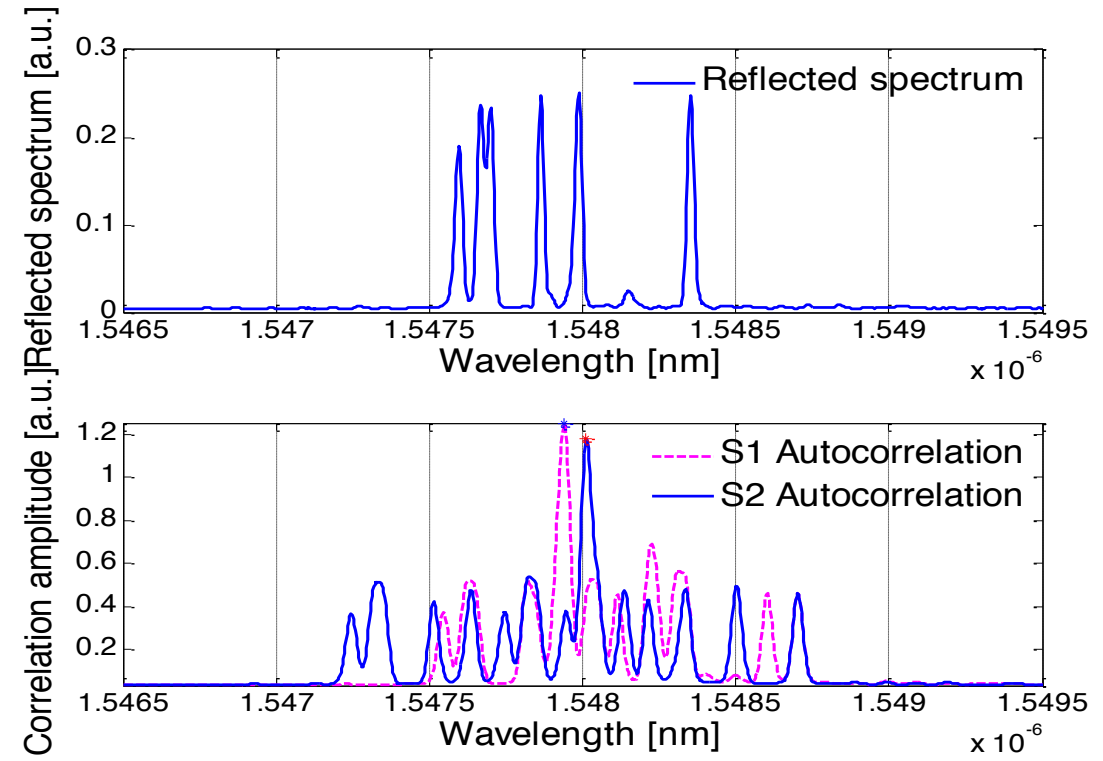

Fig. 11. Readout of the experimental validation, the two encoded sensors are interfering spectrally while the identification function obtains their central position.

The performed experiments confirmed the simulations and validated the detection capability of the system under overlapping conditions. In Fig 12. it is plotted the central wavelength detected for each sensor during the spectral sweep.
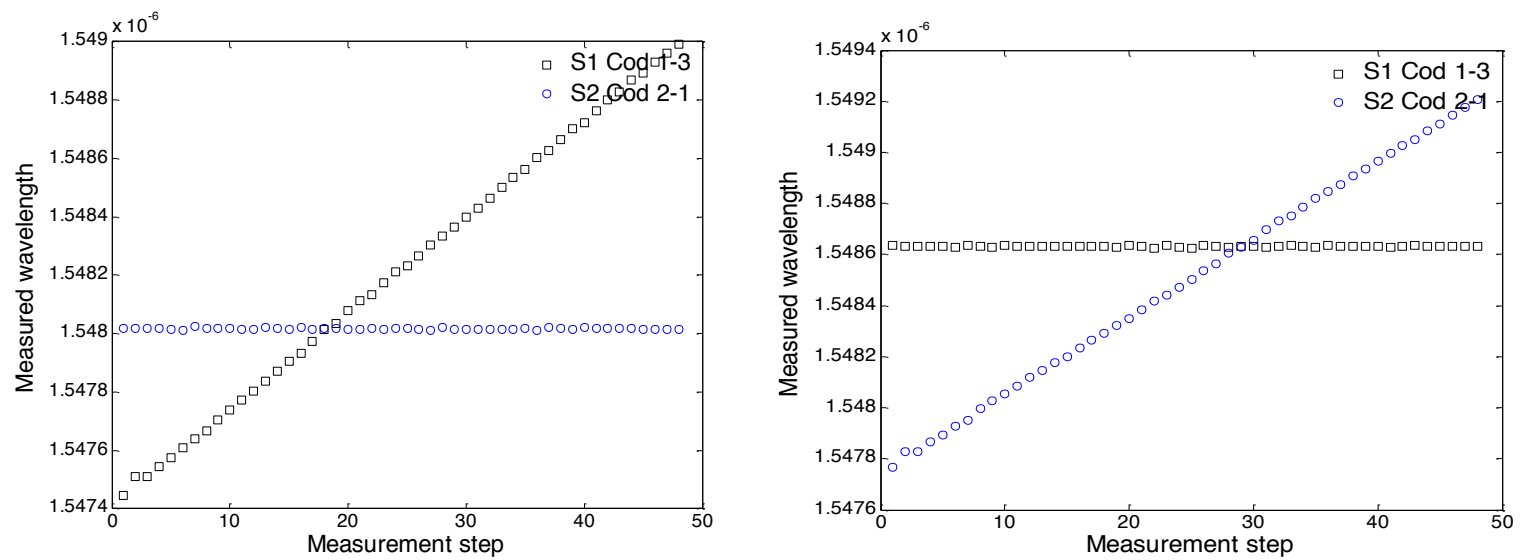

Fig. 12. Detected wavelength of two encoded sensors overlapping spectrally, the plot in the left shows the detected wavelength of sensors S1 and S2 when S1 is being elongated and cross over S2. The right plot shows the sensor S2 crossing spectrally S1. 
In the processing stage, it is performed the demodulation algorithm, which uses the correlation product described in section 2.b. At this stage, every readout from the system is stored in the computer, where the reference - theoretical spectral response of the sensors have been previously charged. After every readout the theoretical codes are swept over the spectrum and the resulting scalar product between the reference sensor and the total readout is stored as the correlation product. Then, it is found the maximum of the correlation product corresponding to the central wavelength of every sensor.

After computing the central position in the spectrum of the 2 overlapping sensors it is possible to find the relative position of each sub-band depending on the codeword used. Thus, we can tell when two sub-bands of different sensors are overlapping. In this case we proceed to re-calculate the central position of each sensor from the unperturbed sub-bands.

The whole demodulation procedure is very simple and fast, very similar for example to a conventional WDM interrogation system. Where, the readout from the system is stored in the processing unit and their peaks are determined by finding the local maxima in the spectrum.

\section{Conclusions}

The design of spectrally encoded FBG sensors, based on orthogonal codes, has been proposed. With this approach, complete identification of the FBG sensor could be achieved even when two sensors are overlapping or crossing each other spectrally. The direct autocorrelation identification algorithm was described and a 'two-steps' improvement was proposed in order to effectively track the measured wavelength from the sensors with zero error.

The implementation of the tracking system was simulated; this allows us to define the appropriate limits for the shape parameters of the encoded sensors. Low reflectivity is needed when using the proposed sensors in serial configurations. The error of the system is directly related to the width of the sub-bands. In this regard, the design of the superimposed-FBGs should be carried out aiming to obtain reflection sub-bands as thin as possible.

The proposed sensors are intended to enhance the number of sensors deployed in an optical sensing network since two orthogonal sensors can work at the same spectral range. In this way it is possible to either have more sensors in the same spectral range or reduce the source bandwidth maintaining the number of sensors, reducing consequently the overall cost of the measurement system; another interesting feature is the association of the unique spectral signature of each sensor with their spatial location or function in a network; in this way, the sensor's spectral shape allows to bring more information into the measurement system.

The main features necessary to the implementation of spectrally encoded fiber Bragg grating sensors are outlined in this work. The manufacturing process was performed achieving very accurate spectral shape for the proposed devices. Validation of the performance of encoded FBG sensors was performed successfully and it was verified the good performance of the sensors under overlapping conditions.

\section{Acknowledgements}

The authors wish to acknowledge the Spanish MCINN support through the projects TEC2013-42332-P, and TEC 2009-12169. 